\title{
Computation and Mutagenesis Suggest a Right-Handed Structure for the Synaptobrevin Transmembrane Dimer
}

\author{
Karen G. Fleming ${ }^{1 *}$ and Donald M. Engelman ${ }^{2}$ \\ ${ }^{1}$ Department of Biophysics, Johns Hopkins University, Baltimore, Maryland \\ ${ }^{2}$ Department of Molecular Biophysics and Biochemistry, Yale University, New Haven, Connecticut
}

\begin{abstract}
Biological membrane fusion involves a highly precise and ordered set of proteinprotein interactions. Synaptobrevin is a key player in this process. Mutagenesis studies of its single transmembrane segment suggest that it dimerizes in a sequence specific manner. Using the computational methods developed for the successful structure prediction of the glycophorin A transmembrane dimer, we have calculated a structural model for the synaptobrevin dimer. Our computational search yields a well-populated cluster of righthanded structures consistent with the experimentally determined dimerization motif. The threedimensional structure contains an interface formed primarily by leucine and isoleucine side-chain atoms and has no interhelical hydrogen bonds. The model is the first three-dimensional picture of the synaptobrevin transmembrane dimer and provides a basis for further focused experimentation on its structure and association thermodynamics. Proteins 2001;45:313-317. $\odot 2001$ Wiley-Liss, Inc.
\end{abstract}

Key words: membrane protein; structure model; synaptobrevin; SNARE

\section{INTRODUCTION}

Since high-resolution structural information of membrane proteins has proven difficult to obtain using conventional techniques, we have pursued a computational approach that couples a restrained modeling protocol with experimental mutational sensitivity in order to generate three-dimensional structures. ${ }^{1-3}$ The restrained modeling strategy is based on a consideration of some of the structural constraints imposed by the membrane upon protein folding, which suggest that the initial formation of a transbilayer $\alpha$-helix can be thermodynamically uncoupled from subsequent intrabilayer helix-helix self-associations. ${ }^{4,5}$ Computationally, this rationale leads to a simplification in structure prediction, since searches for transmembrane helix oligomers can be accomplished by exploring the intermolecular interactions between prefolded $\alpha$-helices. In this manner, a global computational search can be used to find a small number of chemically reasonable models from which a preferred model can be selected using experimental data.

This structure determination strategy has been tested experimentally in the case of the glycophorin A transmembrane segment (TMS) dimer. The restrained computa- tional search we used in this study generated a threedimensional structure consistent with the extensive mutagenesis data of Lemmon et al. ${ }^{6}$ Subsequently, the solution NMR structure of the transmembrane peptide of the glycophorin A TMS in dodecylphosphocholine micelles was solved. ${ }^{7}$ Comparison of the model and the NMR structure reveals a root mean square difference of less than $0.8 \AA$ for backbone atoms over the simulated range. Thus, the computational approach can be used to generate a model that captures the major features present in a three-dimensional structure, representing a body of experimental observations in a framework for further experimental tests.

\section{MATERIALS AND METHODS \\ Sequence and Search}

A sixteen-residue sequence corresponding to residues 97-112 (IILGVICAIILIIIIV) of the full-length synaptobrevin II protein was used in the global search. The simulated annealing search calculations have previously been described in detail. ${ }^{1-3}$ These were carried out following the published protocol except that the molecular dynamics were implemented in $\mathrm{CNS}^{8}{ }^{8}$ and the $\mathrm{OPLS}^{9,10}$ topology and parameter sets were used with all polar hydrogens included. In addition, we optimized the side-chain rotamers of the default starting structure for each helix using the SCWRL algorithm. ${ }^{11}$

The conformations of pairs of synaptobrevin helices were searched with rotation angles $\alpha$ and $\beta$ from $0^{\circ}-360^{\circ}$ for helices A and B, respectively, starting with both leftand right-handed crossing angles, $\Omega$. The sampling step sizes for the rotation angles $\alpha$ and $\beta$ were $45^{\circ}$, and four trials were carried out starting from each conformation using simulated annealing of all atomic coordinates during which rotation- and crossing-angles were free to vary. The resulting 512 structures were analyzed to determine the final crossing angle (either right- or left-handed) as well as to determine the rotation angles, $\alpha$ and $\beta$, of both helices.

Clusters of low-energy structures were calculated by determining the frequency of structures appearing in a particular region of interaction space using a cutoff of $1.0 \AA$

Grant sponsor: NIH; Grant number: GM57534 (to KGF); Grant sponsor: NIH; Grant number: GM54160 (to DME)

*Correspondence to: Karen G. Fleming, Department of Biophysics, Johns Hopkins University, 420 Jenkins Hall, 3400 North Charles

Street, Baltimore, MD 21218. E-mail: karen.fleming@jhu.edu

Received 5 April 2001; Accepted 17 July 2001 


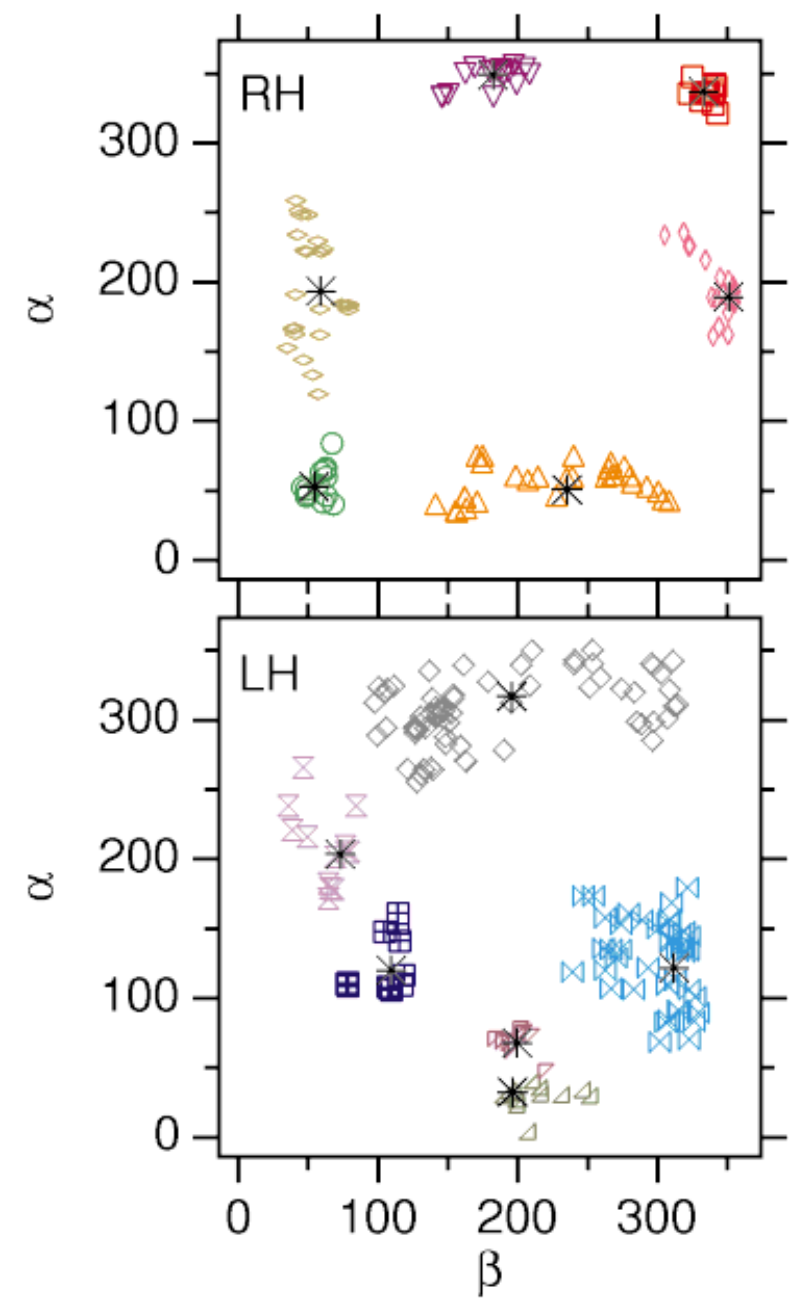

Fig. 1. The frequency of structures obtained by simulated annealing found in $\alpha / \beta$ rotation space. Each cluster is shown in a different color. Black stars represent the positions of the average structure for each cluster. RH: Results for right-handed helical pairs. The green cluster of structures ( $\alpha=60, \beta=60)$ contains the symmetric dimers that are consistent with the experimentally determined dimerization motif. LH: Results for left-handed helical pairs.

root mean square deviation and a minimum requirement of ten structures to define a cluster. The clustering results are shown in Figure 1. Clusters of both right- and lefthanded helices were found; however, only three sufficiently populated regions were consistent with a symmetric dimeric interaction. Two of these are right-handed and one is left-handed. Symmetric dimers are located along the diagonal $\alpha=\beta$ in Figure 1:RH and 1:LH.

An average structure for each cluster was calculated and then evaluated for consistency with the biochemical mutational sensitivity ${ }^{12}$ by inspection of the interhelical interaction energy for each residue calculated using $\mathrm{CNS}^{8}$

\section{Occluded Surface Analysis}

The interchain occluded surface area was calculated using an extension of the OS algorithm. ${ }^{13}$ In this method, a molecular dot surface of each residue is calculated with a $1.4 \AA$ probe using the MS program of Connolly. ${ }^{14} \mathrm{~A}$ normal

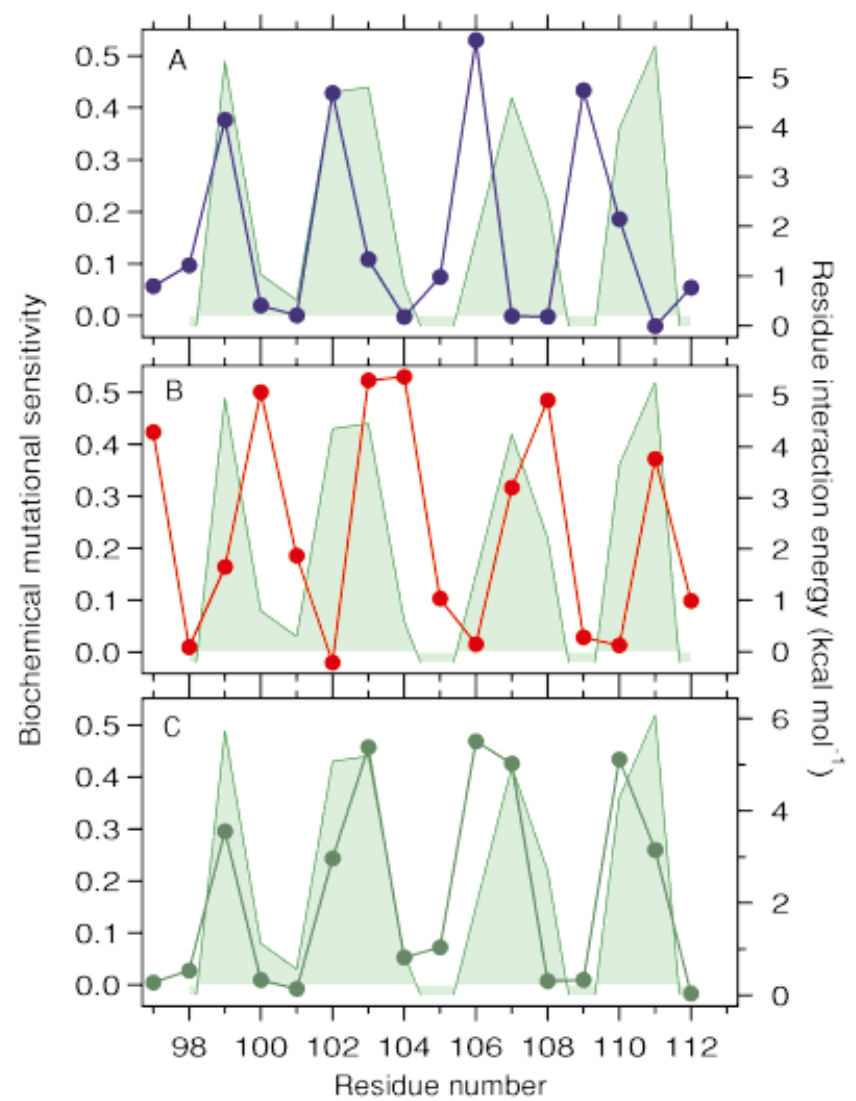

Fig. 2. Correlation between experimental mutational sensitivity and interaction energy. The average mutational sensitivity per residue (where the scale is $[1 \text {-(normalized monomer/dimer ratios) }]^{-1}$ (taken from Laage and Langaos $\mathrm{Ch}^{12}$ ) is overlaid upon the residue interaction energy calculated for average structures representing the blue, red, and green clusters plotted in Figure 1. The green cluster is the preferred model, and subsequent genetic experiments confirm that $\mathrm{L}^{106}$ is important for association. ${ }^{17}$.

is then extended radially from each dot until it either intersects the van der Waals surface of a neighboring atom or reaches a length of $2.8 \AA$ (the diameter of a water molecule). The occluded surface area, OS, is that molecular surface area on the originating atom associated with normals that intersect another atom as opposed to reaching the $2.8 \AA$ limit; all other molecular surface area is considered non-occluded. The interchain occluded surface area is calculated by summing the occluded surface area for only those extended normals that were found to intersect atoms on the opposing chain.

\section{Hydrogen Bonding and Rotamer Distribution and Optimization}

The disulfide and hydrogen bonding potentials of cysteines 103 in the $\mathrm{A}$ and $\mathrm{B}$ chains were evaluate using HBPLUS. ${ }^{15}$ The default cutoff distance for assigning disulfide bridges was used. Evaluation of rotamer distributions was determined using the program BBDEP. ${ }^{16}$ Side-chain rotamer optimization was carried out using the using the SCWRL algorithm. ${ }^{11}$ 


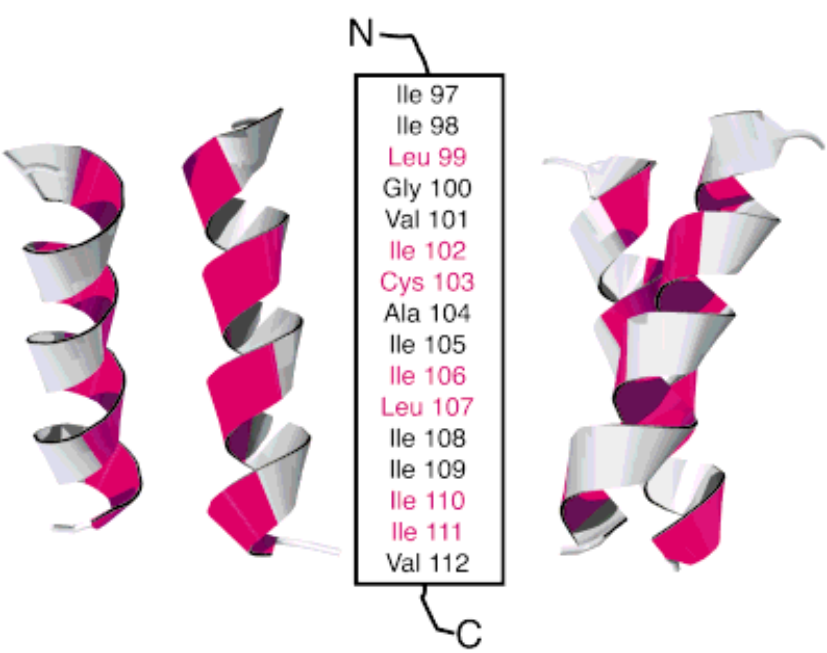

Fig. 3. Overall geometry of the synaptobrevin dimer. An N, $\mathrm{C}^{\alpha}, \mathrm{C}$ ribbons representation of the synaptobrevin dimer is shown from two views. The interacting residues are colored in magenta. Left: View illustrating the symmetric nature of the interaction. Right: Rotated $90^{\circ}$ revealing the crossing angle between the two helices. This image was generated using Deep View and PovRay.

\section{RESULTS AND DISCUSSION Right-Handed Dimer}

Using the methods that have been successful in predicting the structure of the glycophorin A transmembrane dimer, we have predicted an ensemble of dimeric structures for the transmembrane segment of synaptobrevin. A comparison of the residue interaction energy of the symmetric dimeric structures with the SDS-PAGE mutagenesis sensitivity data is shown in Figure 2. This analysis reveals that the interhelical interaction energy of the cluster shown in green [Figs. 2(C) and 1:RH at $\alpha=\beta=60$ ] agrees well with the dimer interface predicted from the experimental mutagenesis data of Langosch and coworkers. ${ }^{12,17}$ Although there appears to be an inconsistency between the biochemical mutagenesis and the computational model at residue Leu ${ }^{106}$ [Fig. 2(C)], subsequent experiments by Laage and coworkers using a genetic assay ${ }^{17}$ confirm that Leu ${ }^{106}$ is important for synaptobrevin self-association, as the computational model predicts. This prediction revealed an important aspect of the modeling showing that it can suggest further experiments.

The experimental data eliminate the other two symmetric dimer structures from consideration. Although the blue cluster does show some consistency with the mutagenesis data, this modeled interaction motif is offset by one amino acid towards the C-terminal end of the helix. Further, the structure represented by the red cluster suggests an interaction surface located on the opposite helical face as compared to the experimental mutational sensitivity.

\section{Synaptobrevin Model}

The ensemble of structures that are consistent with the experimental data include 13 structures. The general organization of the two helices is shown in Figure 3. Analysis of the average structure of the ensemble reveals

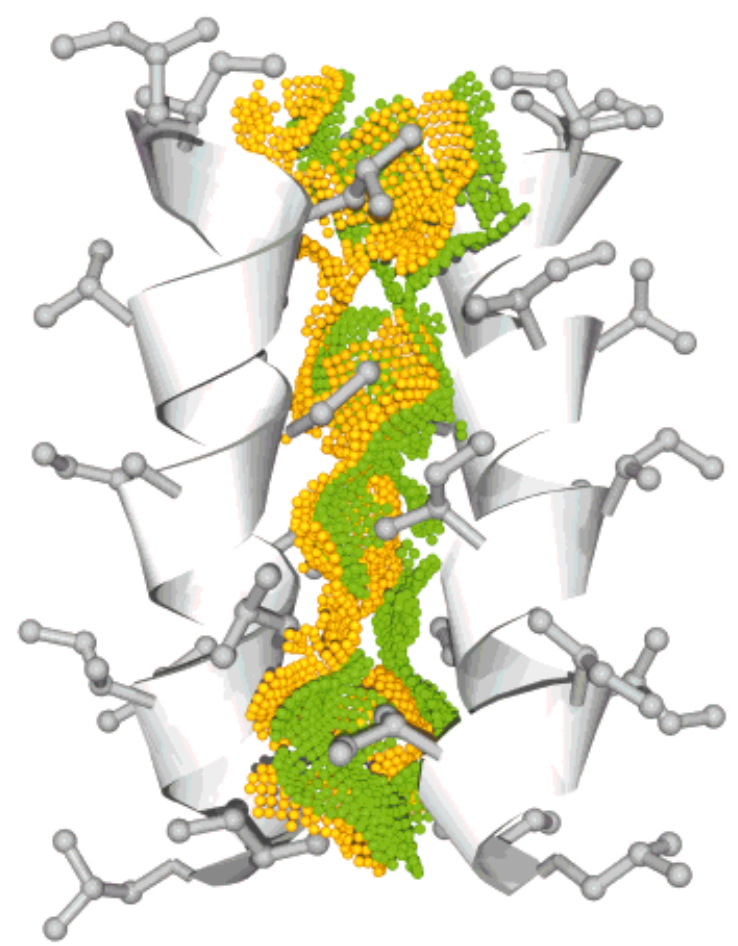

Fig. 4. Interaction surface of the synaptobrevin dimer. A ribbon diagram of the synaptobrevin dimer with side-chain atoms is shown overlaid by the interacting occluded surfaces. The interchain occluded surface of the A and B chains are shown in yellow and green, respectively. This image was generated using Deep View and PovRay.

that the overall geometry is described by an $\alpha$-helix with approximately 3.65 residues per turn. The helices are oriented with respect to each other with a crossing angle of $\Omega=-38^{\circ}$ with an average shift of 0.1 A along the long axis of the helix. The closest approach of the helices to each other occurs at residue 103 where the center-center distance of the helices is $8.6 \AA$. The residues that were found to be sensitive to mutation are pointing toward the dimer interface. As can be seen in Figures 3 and 4, the interaction interface wraps around the helix in a righthanded manner from the $\mathrm{N}$-terminus to the $\mathrm{C}$-terminus. Upon dimerization, $396 \AA^{2}$ of molecular surface area (per dimer) becomes buried (using the MSRoll algorithm ${ }^{14}$ with a probe radius of $1.4 \AA$ ) and $350 \AA^{2}$ (per dimer) becomes occluded (using the OS algorithm ${ }^{13}$ ).

The structure of the cysteine residues in the synaptobrevin dimer are of particular interest since cysteine is the only moderately polar residue found at the dimer interface. Cysteine at position 103 is conserved within most of the synaptobrevin II isoforms. The average distance between the $\mathrm{Cys}^{103} \mathrm{~S}$ atoms on the $\mathrm{A}$ and $\mathrm{B}$ chains is $4.26 \AA$, positioning them outside of the range for prediction of a disulfide bond. ${ }^{15}$ In 12 of the 13 structures in the cluster, the $\mathrm{Cys}^{103} \mathrm{~S}$ atom serves as a hydrogen bond donor for the $\mathrm{Leu}^{99} \mathrm{O}$ on the same chain. The average hydrogen bond length from the donor to the acceptor atom is $2.85 \AA$. Sulfydryl groups that are hydrogen-bonded to carbonyl oxygens at position $i-4$ in $\alpha$-helices commonly occur in soluble proteins. ${ }^{18}$ The $\mathrm{Cys}^{103} \mathrm{~S}$ conformation observed in 
these twelve structures supports this experimental observation that disulfide bond formation is not required for dimerization. In membrane proteins, this hydrogenbonding pattern is observed in the photosynthetic reaction center structure ${ }^{19}$ and in the pentameric ion channel phospholamban. ${ }^{1,20,21}$

In contrast, one of the thirteen structures shows a different hydrogen-bonding pattern. In this case, one of the intrachain $\mathrm{Cys}^{103} \mathrm{~S}-\mathrm{Leu}^{99} \mathrm{O}$ bonds is absent and instead, the $\mathrm{Cys}^{103} \mathrm{~S}$ is positioned just outside of disulfide bonding distance from the $\mathrm{Cys}^{103} \mathrm{~S}$ on the opposing chain. This structure is achieved without large conformational changes to the molecule and is consistent with the possibility that a disulfide bond could be induced under favorable redox potential. This observation initially appeared inconsistent with the experimental data, which lack evidence for disulfide bond formation in membranes in vivo. However, experimental results support the notion that a disulfide bond can occur under oxidizing conditions by treatment with iodine, ${ }^{12}$ and our structure is consistent with this observation. In the absence of large conformational changes, the disulfide bond does not appear possible between dimeric helices in either the red or blue clusters (shown in Figs. 1 and 2), since the cysteines are facing outward.

\section{Specific Packing Drives Dimerization}

The synaptobrevin transmembrane domain includes 5 isoleucines and 2 leucines at the interface in addition to the 3 isoleucine residues not found at the interaction face. This raises the question of how such an apparently nonspecific primary sequence gives rise to a unique dimeric interaction. We used the occluded surface algorithm to evaluate the interhelical packing and to describe the spatial complementarity at the interface. The occluded surface area is related to, but distinct from, the buried molecular surface in that it represents that portion of the molecular surface of an atom that is occluded by its surrounding atoms. It can be thought of as a degree of atomic interaction and interpreted as an estimate of packing. A view of the interchain occluded surface area for each helix is shown in Figure 4. In contrast to the glycophorin A TMS interface, OS analysis of the synaptobrevin structure suggests that side chain atoms, not main chain atoms, are primarily responsible for interhelical contacts in this case. The side chains of $\mathrm{Leu}^{99}, \mathrm{Cys}^{103}$, $\mathrm{Ile}^{106}$, Leu ${ }^{107}$, and $\mathrm{Ile}^{110}$ make the most packing interactions ( $\sim 25 \AA^{2}$ OS per residue per monomer), followed by the side chains of $\mathrm{Ile}^{102}$ and $\mathrm{Ile}^{111}\left(\sim 15 \AA^{2}\right.$ per residue per monomer) and the side chain of $\mathrm{Ile}^{98}$, which makes nominal packing interactions ( $\sim 8 \AA^{2}$ per monomer). The occluded surface distribution is similar in both chains, suggesting a geometry of symmetric packing. The small translational shift in the long axis of the helices with respect to each other (only $0.1 \AA$ ) supports this idea. Figure 4 also shows the detailed geometry and complementarity of the van der Waals interactions possible in this dimer. Specific interactions between the helices are mediated by the side chain of one motif residue packing against those in the opposing chain that are prior to it and that follow it: e.g., the side chain of $\mathrm{Ile}^{102}$ on one helix packs against the side chains of $\mathrm{Leu}^{99}$ and $\mathrm{Cys}^{103}$ on the other. The OS distribution agrees well with the experimental data, since the extensive packing interactions mediated by side chain atoms would be significantly reduced upon mutation of any of the interfacial residues to alanine and would be expected to destabilize the dimerization.

\section{Comparison With the Glycophorin A Dimer}

Although specific packing appears to be providing specificity and stability in both the synaptobrevin and glycophorin A TMS dimers, there are significant differences between the two structures. It is noteworthy that the GV sequence in the synaptobrevin dimer appears not to contribute to its interaction energy, since, in contrast, this portion of the glycophorin A TMS motif plays a key role in its dimerization. A second difference between the two structures is the buried surface and contact area. The total surface area buried upon dimerization is much less for synaptobrevin than for glycophorin (396 $\AA^{2}$ vs. $550 \AA^{2}$ of molecular surface area, $350 \AA^{2}$ vs. $502 \AA^{2}$ of occluded surface area.) The Lennard-Jones interaction energy calculated using CNS and the (same) OPLS parameter set between the synaptobrevin helices is $-34 \mathrm{kcal} \mathrm{mol}^{-1}$ as compared to $-48 \mathrm{kcal} \mathrm{mol}^{-1}$ for the glycophorin A TMS model of Adams and Brunger. ${ }^{2}$

A third potential difference between the two structures is the potential entropic cost of dimerization due to side chain torsion angle freedom of the beta branched side chains. The interacting surface of the glycophorin A TMS contains only three residues with some rotamer freedom in an $\alpha$-helix ( $\mathrm{Leu}^{75}, \mathrm{Ile}^{76}, \mathrm{Thr}^{87}$ ) according to the backbone dependent rotamer library of Dunbrack and Karplus. ${ }^{22}$ Further, the side chain torsion angles in the glycophorin transmembrane domain are only slightly displaced from the ideal values of rotamers seen in helices. ${ }^{16,23,24}$ In contrast, the interacting residues of the synaptobrevin TMS are enriched in residues that have side-chain rotamer freedom in an $\alpha$-helix ( $\mathrm{Ile}^{98}, \mathrm{Leu}^{99}, \mathrm{Ile}^{102}, \mathrm{Ile}^{106}$, $\left.\mathrm{Leu}^{107}, \mathrm{Ile}^{110}, \mathrm{Ile}^{111}\right)$. A first approximation of the difference in the potential entropic cost upon dimerization between these two TMS can be estimated by comparing the number of states for each molecule. We have done this by simply summing the possible rotamers available to these side-chains for which the probability of occurrence is $>10 \%$ (according to the backbone-dependent rotamer library of Dunbrack and Karplus ${ }^{22}$ ). This enumeration yields 36 possible rotamer states for interacting residues of the synaptobrevin TMS compared to 14 possible rotamer states for interacting residues of the glycophorin A TMS. While this analysis is extremely simplified, it does suggest the idea that the TMS of synaptobrevin (ILxxICxxILxxII) is likely to pay a higher cost in side-chain rotamer entropy upon dimerization compared to the glycophorin A TMS (LIxxGVxxGVxxT). A more exhaustive study of the conformational space available to the side chains of both proteins (in both monomeric and dimeric forms) will be required to quantify the differences more carefully. 
In sum, all of the observations arising from comparison of the structures of the glycophorin A dimer to the synaptobrevin dimer are consistent with the biochemical findings indicating that the synaptobrevin TMS interaction energy may not be as favorable as that of the glycophorin TMS. 6,12 Additional quantitative experimentation on both the thermodynamics and structure of the synaptobrevin TMS need to be done to test this idea more rigorously.

\section{Experimental Needs Are Modest}

While the mutational sensitivity data available for the synaptobrevin TMS are modest compared to those for glycophorin A, the alanine scanning mutagenesis data alone are sufficient to distinguish among the symmetric dimeric clusters that were found in the computational search. Indeed, examination of the extensive mutagenesis data for the transmembrane domain of glycophorin shows that the mutational sensitivity to alanine at each position would also be able to distinguish between the computational models generated in the global search. It is important to emphasize, however, that the mutagenesis data should span the length of the interaction surface, since experimental data on only 2 or 3 residues may not have been enough in this case to distinguish between the green and blue clusters (shown in Figs. 1 and 2).

\section{CONCLUSIONS}

In this study, we have combined computational methods with biochemical mutagenesis data in order to generate a three-dimensional model for the synaptobrevin transmembrane dimer. Our computational search yields a wellpopulated cluster of right-handed structures consistent with the experimentally determined dimerization motif. The dimer interface is described by a complementary geometry of packing interactions of side chain atoms primarily from leucine and isoleucine residues. The model represents the first three-dimensional picture of the synaptobrevin transmembrane dimer. As such, it provides a basis for further studies of the chemical principles underlying the relationship between its sequence, structure, and association thermodynamics. PDB files for the ensemble of structures are freely available from the following web site, http://www.jhu.edu/ biophys/fleming/.

\section{ACKNOWLEDGMENTS}

We thank Paul Adams for the helix searching algorithms and for many helpful conversations. We acknowledge grants GM57534 (to K.G.F.) and GM54160 (to D.M.E.) from NIH.

\section{REFERENCES}

1. Adams PD, Arkin IT, Engelman DM, Brunger AT. Computational searching and mutagenesis suggest a structure for the pentameric transmembrane domain of phospholamban. Nature Struct Biol 1995;2:154-162.

2. Adams PD, Brunger AT. Improved prediction for the structure of the dimeric transmembrane domain of glycophorin a obtained through global searching. Proteins 1996;26:257.

3. Adams PD, Brunger AT. Towards prediction of membrane protein structure. In: Heijne GV, editor. Membrane protein assembly. Austin, TX: R.G. Landes Co. 1997. p 97.

4. Popot J-L, Engelman DM. Membrane protein folding and oligomerization: the two-stage model. Biochemistry 1990;29:4032-4037.

5. White SH, Wimley WC. Membrane protein folding and stability: physical principles. Annu Rev Biophys Biomol Struct 1999;28:319365.

6. Lemmon MA, Flanagan JM, Treutlein HR, Zhang J, Engelman DM. Sequence specificity in the dimerization of transmembrane $\alpha$-helices. Biochemistry 1992;31:12719-12725.

7. MacKenzie KR, Prestegard JH, Engelman DM. A transmembrane helix dimer: structure and implications. Science 1997;276:131133.

8. Brünger AT, Adams PD, Clore GM, DeLano WL, Gros P, GrosseKunstleve RW, Jiang JS, Kuszewski J, Nilges M, Pannu NS, Read RJ, Rice LM, Simonson T, Warren GL. Crystallography and NMR system: a new software system for macromolecular structure determination. Acta Cryst D 1998;54:905-921.

9. Jorgensen WL, Tirado-Rives J. The OPLS potential functions for proteins. energy minimizations for crystals of cyclic peptides and crambin. J Am Chem Soc 1988;110:1657-1666.

10. Tirado-Rives J, Jorgensen WL. Molecular dynamics of proteins with the opls potential functions simulation of the third domain of silver pheasant ovomucoid in water. J Am Chem Soc 1990;112: $2773-2781$.

11. Bower MJ, Cohen FE, Dunbrack RL. Sidechain prediction from a backbone-dependent rotamer library: a new tool for homology modeling. J Mol Biol 1997;267:1268-1282.

12. Laage R, Langosch D. Dimerization of the synaptic vesicle protein synaptobrevin (vesicle-associated membrane protein) II depends on specific residues within the transmembrane segment. Eur J Biochem 1997;249:540-546.

13. Pattabiraman N, Ward KB, Fleming PJ. Occluded molecular surface: analysis of protein packing. J Mol Recog 1995;8:334-344.

14. Connolly ML. Computation of molecular volume. J Am Chem Soc $1985 ; 107: 1118-1124$.

15. McDonald K, Thornton JM. Satisfying hydrogen bonding potential in proteins. J Mol Biol 1994;238:777-793.

16. Dunbrack RL, Cohen FE. Bayesian statistical analysis of protein side-chain rotamer preferences. Prot Sci 1997;6:1661-1681.

17. Laage R, Rohde J, Brosig B, Langosch D. A conserved membranespanning amino acid motif drives homomeric and supports heteromeric assembly of presynaptic SNARE proteins. J Biol Chem 2000;275:17481-17487.

18. Gregoret L, Rader S, Fletterick R, Cohen F. Hydrogen bonds involving sulfur atoms in proteins. Proteins 1991;9:99-107.

19. Diesenhofer J, Michel H. Nobel lecture, The photosynthetic reaction centre from the purple bacterium Rhodopseudomonas viridis. EMBO J 1989;8:2149-2170.

20. Arkin IT, Rothman M, Ludlam CF, Aimoto S, Engelman DM, Rothschild KJ, Smith SO. Structural model of the phospholamban ion channel complex in phospholipid membranes. J Mol Biol 1995;248:824-834.

21. Arkin IT, Adams PD, Brunger AT, Aimoto S, Engelman DM, Smith SO. Structure of the transmembrane cysteine residues in phospholamban. J Membr Biol 1997;155:199-206.

22. Dunbrack RL, Karplus M. Backbone-dependent rotamer library for proteins: application to side-chain prediction. J Mol Biol 1993;230:543-574

23. MacKenzie KR. Structure determination of the dimeric membrane spanning domain of glycophorin A in detergent micelles by triple resonance nuclear magnetic resonance spectroscopy, in molecular biophysics and biochemistry. New Haven: Yale University. 1996. p 1-349.

24. Ponder JW, Richards FM. Tertiary templates for proteins. Use of packing criteria in the enumeration of allowed sequences for different structural classes. J Mol Biol 1987;193:775-791. 